\title{
GROUP THEORY REVIEWS
}

\author{
IN THE \\ JAHRBUCH ÜBER DIE FORTSCHRITTE DER MATHEMATIK
}

BY PROFESSOR G. A. MILLER.

A few erroneous statements found in a recent volume of the JAhrbuCH ÜBER dIe Fortschritte DER MathematiK and relating to the theory of groups led the present writer to make a brief survey of the other volumes of this series for the purpose of determining the reliability of the reviews relating to this particular subject. As these reviews are so widely read and so frequently referred to, it seems desirable to note here a few of the instances of inaccurate or misleading statements found therein, since some of these instances may be instructive and since such a notice may tend to prevent a repetition of these particular errors. The fact that a few of the many reviewers failed to maintain the high standards of this classic series should not be surprising, and a knowledge of their shortcomings can only increase the usefulness of this series, which has had no serious competitor, since its inauguration about half a century ago, in its field of providing critical reviews of the entire current mathematical literature relating to important advances.

The erroneous statements mentioned above are found on page 164 of volume 44 (1918) and relate to the possible sets, or systems, of independent generators of a finite group whose order is a power of a prime. It is there stated that the number of the operators in such a set is equal to the index of the commutator subgroup. In the following sentence the equally incorrect statement is made that a system of independent generators of such a group can be obtained by taking one operator from each of the co-sets with respect to the commutator subgroup. According to these statements the cyclic group of order $p^{m}, p$ being a prime number, would have a set of $p^{m}$ independent generators, since the commutator subgroup of this group is the identity. On the contrary, it is well known that only one operator can appear in such a set of generators.

These statements are so obviously incorrect that one might be inclined to attribute them to a harmless and amusing over- 
sight if they were not followed by a sentence which is not only incorrect but also practically meaningless. In that sentence, it is stated that the author in question considered the series of commutator subgroups and made assertions about their minimal order. As a matter of fact minimal orders of the centrals of these commutator subgroups were considered in the article under review. From what has been noted, it is evident not only that the reader of this particular review will get very little knowledge therefrom regarding the significance of the article in question, but that he is also in danger of being led to regard a theorem as true which he will find later to be false.

It may be of interest to note in this connection that each one of the published volumes of the JAHRBUCH contains reviews of articles on group theory and that more than thirty different men wrote these reviews. Most of these men published only a few such reviews. Among the early reviewers along this line E. Netto stands out most prominently for his long service and the number of articles he reviewed. In particular, Netto reviewed most of C. Jordan's articles on this subject as well as his now classic Traité des Substitutions, 1870. These reviews of Jordan's work were not always fair. For instance, in the review of this Traité, Netto said, "there are still many gaps to be filled, much diffuseness to be removed, and many proofs to be corrected."

The fact that Jordan was not pleased with this review is clear from a note published in volume 79 of CRELLE (p. 258), where Jordan refers to the first publication of Netto in support of the criticism quoted above, and says that Netto was not entirely successful in his effort. Jordan adds that he could not hope that his Traité des Substitutions was free from inexact statements in view of its great extent and of the novelty and difficulty of the subject treated, but that he was convinced that there were few such statements; he himself had found two which he corrected promptly and he would be greatly obliged to those who would point out others.

In a review which appeared on page 41 of the first volume of the JAHRBUCH, Netto makes an incorrect statement relating to a fundamental question, and hence it may be worth noting here. After defining the term isomorphic group in accord with our present definition of simply isomorphic group, Netto considers $n$ rational functions $F_{1}, F_{2}, \cdots, F_{n}$ of the $m$ variables $x_{1}$, 
$x_{2}, \cdots, x_{m}$, which constitute a complete set of conjugates under a substitution group $G$ on these $m$ variables. He observes correctly that the substitutions of $G$ transform $F_{1}$, $F_{2}, \cdots, F_{n}$ according to a transitive substitution group, but he adds incorrectly that this transitive group is isomorphic (simply isomorphic) with $G$. It is now well known that a necessary and sufficient condition that the transitive group on the $F$ 's be simply isomorphic with $G$ is that the subgroup composed of all the substitutions of $G$ which transform into itself one of these $F$ 's should be non-invariant under $G$ and should not include any invariant subgroup of $G$ besides the identity.

An article of unusual importance in the development of group theory is the one in which A. Capelli introduced the concept of general isomorphisms between two groups. It is therefore of interest to note here several inaccuracies which appear in the review of this article, published on page 106 of volume 10 of the JAHRBUCH. Near the beginning of this review the term subgroups is used to denote co-sets, and the definition of general isomorphisms is obscured thereby. Near the end of the review the statement that a transitive group of order $p$ and of degree $n>p$ cannot be primitive is found. It was known even at the time of A. L. Cauchy that the order of a transitive group is always divisible by its degree and that therefore there can be no transitive group of order $p$ and of degree $n>p$. In the same review, theorems relating to Sylow subgroups, which were well known at a much earlier date, were spoken of as if they were new.

On page 116 of volume 16 it is stated that in groups of order $n=p^{\alpha} \cdot q^{\beta} \cdots$, where $p, q, \cdots$ are prime numbers, which contain only one subgroup of each of the orders $p^{a}$, $q^{\beta}, \cdots$, all the substitutions are commutative. On the following page, it is said that these groups are the only ones in which every subgroup is invariant. As a matter of fact the non-abelian groups in which every subgroup is invariant were investigated much later and were named Hamiltonian groups by $\mathrm{R}$. Dedekind. It is true that each of these groups contains only one Sylow subgroup for every prime which divides the order of the group, but this is not a sufficient condition for a Hamiltonian group. It is fortunate that the statement just noted was not accepted as true since otherwise the interesting later investigations relating to Hamiltonian groups would probably not have been made. 
It is interesting to note that some of the most important articles were reviewed very briefly. For instance, Netto devoted only eight lines to the review of the important article by L. Sylow which appeared in volume 5 of the MathematIsche Annalen. A special case of a fundamental result contained in this article but which Netto failed to mention was later credited to Netto in a review written by F. Meyer and published on page 139 of volume 20 of the JAHRBUCH. The latter review is such a mixture of meaningless statements and trivialities that it would be almost amusing if it did not appear in a work which maintains, on the whole, very high standards.

In view of the fact that most of the reviewers for the JAHRBUCH have been more familiar with the work of German authors than with that of authors of other lands, it is only natural that one sometimes finds undue credit given to the former. This does not imply that these reviewers were conscious of any unfairness in giving credit. An interesting example of this apparently unconscious bias is found in the review of an article by the present writer, on page 98 of volume 27 . It is there stated that the theorem that every group of order $p^{a}, p$ being a prime number and $\alpha>3$, contains an abelian subgroup of order $p^{3}$, is a consequence of theorems due to Hölder and Frobenius. As a matter of fact Hölder needed this theorem but failed to find it when he was determining the groups of order $p^{4}$, and he devoted more than 30 pages of the Mathematische AnNalen (vol. 43) to a discussion of properties of these groups. When the present writer communicated this theorem to Hölder, the latter replied that after receiving this information he at once went over his groups of order $p^{4}$ and verified that each of them actually contains an abelian subgroup of order $p^{3}$. $\mathrm{He}$ realized that the use of this theorem would have greatly simplified his discussions. The theorem might be said to be a consequence of developments due to Sylow rather than of work done by Hölder and Frobenius.

UNIVERSITY OF ILLINOIS, December 22, 1920. 